\section{Jogos recreativos melhoram os fatores de risco cardiovascular em crianças e adolescentes com dislipidemia e obesidade abdominal: um estudo piloto}

\author{
Recreational games improve \\ cardiovascular risk factors in children \\ and adolescents with dyslipidemia and \\ abdominal obesity: a pilot study
}

Nilton Rosini $i^{1,2}$

Rodrigo Diegoli Rosini²

Elisa Cristina Vidotto Bruns ${ }^{3}$

Grasiele Demarche Camillo ${ }^{3}$

Marcos José Machado ${ }^{4}$

Edson Luiz da Silva ${ }^{1,4}$

\section{RESUMO}

O objetivo do presente estudo piloto foi verificar se a prática de jogos recreativos e cooperativos promove modificação dos fatores de risco cardiometabólicos em crianças e adolescentes. Vinte e seis estudantes ( 15 meninos), com idade entre oito e 12 anos, portadores de dislipidemias e/ou obesidade abdominal participaram desse estudo. As atividades recreativas e de lazer foram realizadas $2 \mathrm{~h} / \mathrm{dia}$, três vezes por semana, durante 3,5 meses, e foram supervisionadas por profissional de Educação Física. No início e no final do estudo, amostras de sangue foram coletadas (jejum 12-14 h) para as análises bioquímicas. Além disto, foram realizadas medidas antropométricas por métodos padronizados. De acordo com os resultados, houve melhoras significativas na concentração de HDL-colesterol (+3,7\%), triglicérides (-25,4\%), índice de Castelli I (-7,1\%), ácido úrico (-15,4\%), circunferência da cintura $(-5,4 \%)$ e relação circunferência da cintura/altura $(-4,3 \%)$. Pode-se concluir que a prática de jogos recreativos e cooperativos promoveu melhoras cardiometabólicas, particularmente nos parâmetros lipídicos e na obesidade central, nas crianças e adolescentes com dislipidemias e/ou obesidade abdominal que participaram desse estudo. Sugere-se, assim, que a atividade física na criança e no adolescente, visando à prevenção dos fatores de risco para as doenças cardiovasculares, não requer a adoção de treinamento intensivo de exercícios físicos que exijam a capacidade máxima do praticante.

\section{PALAVRAS-CHAVE}

Crianças, Adolescentes, Lipoproteínas, Dislipidemia, Atividade Motora, Obesidade.

\begin{abstract}
The aim of this pilot study was to verify whether the practice of recreational and cooperative games promotes the reduction of cardiometabolic risk factors in children and adolescents. Twenty-six students (15 boys), aged 8-12 y, with dyslipidemia and/or abdominal obesity, participated of this study. Recreational and leisure activities were performed $2 \mathrm{~b}$ daily, 3 times a week, during 3.5 months, and they were supervised by physical educator. At the beginning and end of the study, blood samples (12-14 b fast) were withdrawn for the measurement of biochemical parameters. Furthermore, anthropometric measures were assessed using standard procedures. According to results, there were significant improvements in the levels of HDL-cholesterol (+3.7\%), triglycerides (-25.4\%), Castelli I index $(-7.1 \%)$, uric acid $(-15.4 \%)$, waist circumference (-5.4\%) and waist circumference/height ratio $(-4.3 \%)$. We may conclude that the practice of recreational and cooperative games promoted cardiometabolic improvements in the children and adolescents with dyslipidemias and/or abdominal obesity who participated of this study. It is suggested, therefore, that physical activity in children and adolescents aiming the prevention of risk factors for cardiovascular diseases, does not require the adoption of intensive training exercise with the maximum capacity of the practitioner.
\end{abstract}

\section{KEYWORDS}

Childhood, Adolescents, Lipoproteins, Dyslipidemia, Motor activity, Obesity.
Rev Bras Ativ Fis Saúde p. 121-132 DOI

http://dx.doi.org/10.12820/rbafs.v.19n1p121

1Programa de Pós-Graduação em Farmácia, Centro de Ciências da Saúde da Universidade Federal de Santa Catarina, Florianópolis-SC, Brasil.

2Gerência de Saúde -Secretaria de Estado do Desenvolvimento Regional em Brusque - $16^{a}$ SDR - Brusque-SC, Brasil;

3Secretaria de Educação - Prefeitura Municipal de Guabiruba-SC, Brasil.

4Departamento de Análises Clínicas, Centro de Ciências da Saúde, Universidade Federal de Santa Catarina, Florianópolis-SC, Brasil.

Childbood, Adolescents, Lipoproteins, Dyshpidemia, 


\section{INTRODUÇÃO}

As previsões para 2015 são de 20 milhões de mortes anuais relacionadas às doenças cardiovasculares (DCVs), sendo que destas, $80 \%$ podem ser prevenidas ${ }^{1}$. O processo de urbanização vem condicionando mudanças de comportamento na dieta e na prática de exercícios físicos, favorecendo precocemente o desenvolvimento de fatores de risco para as DCVs. Dentre eles, destacam-se o sobrepeso e a obesidade, a hipertensão, as dislipidemias e o diabetes melito tipo $2^{1}$.

As manifestações clínicas das DCVs tendem a surgir a partir da meia-idade, porém o processo envolvido em sua gênese, como a aterosclerose, com o aparecimento de estrias gordurosas na camada íntima da aorta, pode surgir aos três anos de idade, e nas artérias coronárias durante a adolescência ${ }^{2}$. Além disto, muitos dos fatores de risco presentes no adulto, particularmente o excesso de peso e as dislipidemias, tiveram origem na infância ${ }^{3}$. Kwiterovich $^{3}$ alertou para a presença precoce destes fatores de risco modificáveis em crianças, os quais podem permanecer até a fase adulta, tornando os indivíduos mais propensos ao desenvolvimento da aterosclerose. Dessa forma, encontra-se bem documentada que a melhor estratégia para a redução das DCVs consiste, principalmente, na diminuição dos fatores de risco que participam no desenvolvimento desta desordem. Segundo a I Diretriz de Prevenção da Aterosclerose na Infância e Adolescência ${ }^{4}$, encontra-se consolidado o conceito de que esses tratamentos devem começar na infância. Recentemente, Chacra e Santos ${ }^{5}$ também enfatizaram que estudos observacionais de longo prazo, apresentaram evidências de que a prevenção primária deve ser iniciada na infância e na adolescência.

Dentre as estratégias para a diminuição das DCVs em adultos encontra-se, primeiramente, a mudança no estilo de vida, caracterizada pela prática regular de exercícios físicos - normalmente estruturados e acompanhados por profissionais de saúde - e mudança na alimentação ${ }^{6}$. Na infância e adolescência, estas abordagens também costumam vir acompanhadas de melhorias sociais e comportamentais, ao proporcionarem perda de peso e aumento da autoestima nos indivíduos ${ }^{4}$. Assim, é plausível acreditar que, se os fatores de risco cardiovasculares presentes na infância tendem a permanecer na idade adulta, a melhora destes mesmos fatores ainda na infância contribuirá para a ocorrência de adultos com menores riscos cardiovasculares. Além disto, é importante estabelecer se brincadeiras e jogos realizados no convívio escolar são suficientemente benéficos para a melhoria dos fatores de risco cardiovasculares. Dessa forma, o objetivo do presente estudo foi verificar o efeito da prática continuada de atividades físicas, constituídas por jogos recreativos e cooperacionais, nos fatores de risco para as DCVs em crianças e adolescentes com dislipidemias e/ou obesidade abdominal do município de Guabiruba-SC.

\section{MÉTODOS}

\section{Delineamento do Estudo}

O presente estudo piloto foi do tipo quase experimental e prospectivo (antes e depois). O estudo foi constituído por amostra de conveniência voluntária, não selecionada aleatoriamente e sem cálculo de tamanho mínimo, para a qual foi oferecida a prática de atividades físicas e de mensuração de variáveis antropo- 
métricas e de lipídeos séricos. Os estágios pubertários dos participantes não foram avaliados devido à recusa dos participantes.

\section{População do Estudo}

Para este estudo, foram convidados os alunos da rede municipal de ensino do município de Guabiruba-SC que participaram anteriormente do Projeto Águia (Avaliação Guabirubense na Infância e Adolescência), o qual teve como objetivo identificar a presença de fatores de risco para as DCVs nesta população ${ }^{7,8}$.

A amostra foi obtida por conveniência e selecionada de forma intencional, de modo a atender os critérios de presença de dislipidemias e/ou obesidade abdominal. O grupo de estudo foi constituído de 26 indivíduos (15 meninos e 11 meninas), com idade entre oito e 12 anos (média = 9,7; desvio padrão = 1,4 anos) e pertencentes a diferentes comunidades do município de Guabiruba-SC. Os participantes apresentavam dislipidemias (colesterol total > $150 \mathrm{mg} / \mathrm{dL}$, triglicerídeos $>100 \mathrm{mg} / \mathrm{dL}$, LDL-colesterol $>100 \mathrm{mg} / \mathrm{dL}$ e/ou HDL-colesterol < $45 \mathrm{mg} / \mathrm{dL}$, segundo a I Diretriz de Prevenção da Aterosclerose na Infância e Adolescência ${ }^{4}$ e/ou obesidade abdominal com valores da circunferência da cintura acima do ponto de corte estabelecido por Taylor et al. ${ }^{9}$ e estratificado por sexo e idade. Os estudantes não utilizavam terapia medicamentosa e estavam aptos para as atividades escolares regulares, incluindo a prática esportiva curricular.

O estudo foi desenvolvido em conformidade com os princípios contidos na Declaração de Helsinque (Resolução 466/2012 do Conselho Nacional de Saúde) e foi aprovado pelo Comitê de Ética para Pesquisa em Seres Humanos na Universidade Federal de Santa Catarina ( $\left.n^{\circ} 210\right)$. Todos os participantes apresentaram o Termo de Consentimento Livre e Esclarecido assinado por seus pais ou responsáveis legais.

\section{Atividades Físicas}

As atividades físicas foram realizadas em período extraclasse, com duração de 2 h, três vezes por semana, durante 3,5 meses (105 dias), com a supervisão e acompanhamento de professores de Educação Física. As atividades físicas consistiram em alongamento (5 $\mathrm{min}$ ) e caminhadas/trote (20 min), seguido de circuito de cinco estações ( $35 \mathrm{~min}$ ) e intervalo de $15 \mathrm{~min}$ para lanche composto por sucos e frutas. Jogos recreativos diversos (maremoto, pega-pega com bola, não caia no oceano, etc.) foram praticados por $45 \mathrm{~min}$.

Durante os meses de agosto e setembro, as atividades desenvolvidas na forma de circuito consistiram de movimentos rotatórios com bambolês nos braços ( $1^{\text {a }}$ estação); pular corda ( ${ }^{\text {a }}$ estação); movimentos de flexão e extensão de braços, com halteres ( $3^{a}$ estação); saltos sobre elásticos ( $4^{a}$ estação) e; polichinelo ( $5^{\text {a }}$ estação). Os participantes permaneceram em cada estação por um minuto e repetiram todo o circuito cinco a sete vezes. Nos meses de outubro e novembro, as atividades na forma de circuito foram pular corda ( $1^{\text {a }}$ estação); fazer abdominais ( $2^{\mathrm{a}}$ estação); movimentos alternados de pernas ( $3^{\mathrm{a}}$ estação); elevação lateral com halteres ( $4^{\mathrm{a}}$ estação) e; polichinelo ( $5^{\mathrm{a}}$ estação).

A atividade recreativa "maremoto" teve como objetivo estimular a lateralidade, o raciocínio lógico e rápido e o trabalho em equipe. Nessa brincadeira, os participantes foram distribuídos em duas equipes. Os alunos sentaram-se no chão em duas filas ou colunas, com as pernas afastadas e com os braços er- 
guidos e seguiram os comandos do(a) professor(a). Por exemplo, ao comando "onda para direita", os participantes deveriam levar os braços para o seu lado direito; "onda para esquerda", deveriam estender os braços para a esquerda; "onda para frente" ou "onda para trás", os participantes deveriam inclinar o corpo e os braços para frente ou para trás, respectivamente. Quando o professor falava "maremoto" os alunos deveriam se levantar rapidamente e trocar de lugar com os colegas da outra coluna.

$\mathrm{Na}$ brincadeira "pega-pega com bola", o objetivo foi praticar a agilidade. Nessa atividade, um aluno iniciava o jogo com a bola e era o pegador, enquanto os demais eram os fugitivos e ficavam em movimento dentro do espaço físico destinado. $\mathrm{O}$ pegador deveria tocar com a bola nos fugitivos, não valia jogar a bola ou arremessa-la, e o participante que era tocado seria o próximo pegador.

O objetivo da atividade recreativa "não caia no oceano" foi praticar o trabalho em equipe, treinar a agilidade, a coordenação motora ampla e o equilíbrio. Nessa atividade, o grupo de participantes foi distribuído em equipes com quatro alunos, sendo que cada equipe recebeu dois colchonetes. Os alunos deveriam ficar dispostos sobre um colchonete e, ao sinal do professor, deveriam passar para o outro colchonete, sem pisar no chão. A equipe vencedora deveria fazer o percurso em menor tempo. Se um membro da equipe pisasse no chão, todos deveriam voltar para a linha de partida e reiniciar a brincadeira.

A atividade "pega rabo" ou "arranca rabo" teve como objetivo promover a agilidade. Nessa brincadeira, cada aluno recebeu um colete, o qual foi preso em sua bermuda para ser usado como rabo. Ao sinal do professor, eles deveriam proteger o seu "rabo" e, ao mesmo tempo, tentar roubar o "rabo" dos demais. Quem perdesse o "rabo" sairia da brincadeira e entregaria todos os "rabos" que possuísse. Vencia quem, ao final, permanecesse com o seu rabo. O objetivo da brincadeira "pega tudo" também foi treinar a agilidade. Delimitou-se um espaço físico para a atividade e um aluno ficou no centro. Esse aluno foi o "pegador" e os demais ficaram nas laterais e agiram como "fugitivos". Ao sinal do professor, os alunos fugitivos deveriam correr em direção à outra extremidade do espaço físico, sem que o pegador os impedisse. Quem fosse pego passava a ser o pegador. A brincadeira terminava quando todos os alunos fossem pegos.

Na brincadeira "nunca três", o objetivo foi estimular o trabalho em equipe, a agilidade, a velocidade e a matemática. Nessa atividade, um aluno começou sendo o "pegador", enquanto os demais deveriam fugir. Quem fosse pego deveria seguir de mãos dadas com o pegador e assim que estivessem em três, o grupo se dividiria, sendo dois "pegadores" e um "fugitivo". A brincadeira continuava, porém, nunca permanecendo três alunos como "pegadores". A atividade recreativa denominada "nunca quatro" é semelhante à brincadeira "nunca três", alterando-se somente o número de pegadores.

No "jogo dos dez passes", o objetivo também foi estimular o trabalho em equipe, a agilidade, a velocidade e a matemática. Nessa atividade, os participantes foram distribuídos em duas equipes. Enquanto os alunos de uma equipe executavam passes de bola com as mãos em sequência de um a 10, os demais alunos deveriam tentar interceptar a bola, ou os passes, para que eles mesmos pudessem iniciar uma nova sequência de passes. A equipe que conseguisse executar 10 passes consecutivos marcava um ponto. A bola não poderia ser arrancada das mãos do colega, bem como não se podia agarrar o colega para conseguir a bola. 
A atividade denominada "pular o rio" tinha como objetivo treinar a velocidade, a flexibilidade e a agilidade dos participantes. Nessa brincadeira, delimitou-se um espaço para que os alunos executassem saltos em distância. Após todos os participantes terem executado o salto, aumentava-se a distância. O jogo seguiu até que um participante saltasse a maior distância.

Na brincadeira "chutes a gol”, o objetivo foi treinar a força, a direção e a lateralidade. Determinou-se um aluno (ou mais) para ser o goleiro e os demais participantes executavam chutes ao gol, primeiro com a perna direita e, depois, com a perna esquerda. A atividade "vamos driblar e chutar?" teve como objetivo estimular a agilidade, a lateralidade e a velocidade, semelhante à brincadeira "chutes a gol". Porém, antes de executar o chute os alunos deveriam driblar um cone ou um aluno que fazia o papel de zagueiro e, na sequência, efetuavam o chute.

Em relação à intensidade das atividades, no presente estudo piloto foram adotados os critérios de classificação do Department of Health and Human Service (DHHS), contidos no Physical Activity Guidelines for Americans ${ }^{10}$ e citados pelo Centers for Disease Control and Prevention, no School Healthy Guidelines to Promote Healthy Eating and Physical Activity ${ }^{11}$. Para as atividades aeróbias, levou-se em conta o conceito de intensidade relativa (ou nível relativo de esforço), que se baseia no nível individual de condicionamento cardiorrespiratório $^{10,11}$. Em resumo, numa escala de 0 a 10 , onde permanecer sentado equivale a 0 e o mais alto nível de esforço possível representa 10, atividade de intensidade relativamente moderada equivale a 5 ou 6 e o indivíduo percebe que seus batimentos cardíacos e a sua respiração estão mais rápidos do que o normal. A atividade de intensidade vigorosa está no nível 7 ou 8, onde o indivíduo percebe os batimentos cardíacos muito mais rápidos e a respiração muito mais intensa do que os normais ${ }^{10,11}$. Além de atividades aeróbias, foram incluídas no programa atividades de fortalecimento muscular e ósseo, bem como de flexibilidade, agilidade, velocidade e equilíbrio, conforme a recomendação do $\mathrm{DHHS}^{10}$. As atividades foram aplicadas inicialmente em agosto/2011 e cada indivíduo foi observado para atingir um nível de esforço de intensidade moderada. Progressivamente, foi-se aumentando a intensidade dos mesmos exercícios, mantendo-se o mesmo espaço de tempo, até atingirem a classificação de moderado/vigorosa ao final do período (dezembro/2011) $)^{10,11}$.

\section{Coleta de Dados}

No início e ao final do estudo, após jejum de 12-14 h, foram realizadas coletas de sangue da veia antecubital mediana $(10 \mathrm{~mL})$ utilizando-se sistemas a vácuo (Vacutainer-BD, Franklin Lakes-NJ, EUA) em tubos contendo a mistura EDTA-Fluoreto de sódio ou em tubos sem anticoagulantes. Os tubos foram centrifugados imediatamente $(1.500 \mathrm{x} \mathrm{g}, 10 \mathrm{~min})$ e o plasma e o soro foram congelados a $-20{ }^{\circ} \mathrm{C}$ para posterior análise bioquímica. Além disto, foram aferidas as medidas antropométricas (peso, altura, circunferência da cintura e dobras cutâneas) e a pressão arterial dos participantes.

A quantificação dos parâmetros bioquímicos foi realizada de acordo com as metodologias laboratoriais de rotina, por profissionais que não conheciam a procedência das amostras (estudo cego), no Laboratório ROSINI - Análises Clínicas Ltda, anexo ao Hospital Dom Joaquim de Brusque-SC e no Laboratório do Hospital Universitário da Universidade Federal de Santa Catarina, 
em Florianópolis-SC. A glicose foi determinada em plasma fluoretado utilizando-se o sistema de reação glicose oxidase/peroxidase. O colesterol total (CT), o colesterol da lipoproteína de alta densidade (HDL-c), os triglicerídeos (TG) e o ácido úrico também foram quantificados utilizando-se regentes com base na reação de Trinder (oxidase/peroxidase) em sistema automatizado (BTS 370 BioSystms - Connecticut-EUA). A insulina e a proteína C reativa de alta sensibilidade (PCR-as) foram quantificadas no soro por meio do sistema Immulite 2000 (Siemens Healthcare - Reino Unido).

Os seguintes parâmetros foram obtidos por cálculo: resistência à insulina (Homeostatic model assessment of insulin resistance index; $\mathrm{HOMAi}=$ insulina sérica de jejum $(\mu \mathrm{U} / \mathrm{mL})$ x glicose sérica de jejum $(\mathrm{mg} / \mathrm{dL}) / 405)^{12}$; colesterol da lipoproteína de baixa densidade (LDL-c): $[\mathrm{LDL}-\mathrm{c}=\mathrm{CT}-(\mathrm{TG} / 5+\mathrm{HDL}-\mathrm{c})]^{13}$; não-HDL-c = CT - HDL-c; Índice de Castelli I = CT/HDL-c e; Índice de Castelli II = LDL-c/HDL-c ${ }^{14}$.

Os parâmetros antropométricos foram aferidos por meio das medidas de peso (P), em kg, e altura (Alt), em metros (m), com equipamento Welmy constituído de balança com capacidade para até $200 \mathrm{~kg}$ e escala de $100 \mathrm{~g}$ e estadiômetro de capacidade para até $2,0 \mathrm{~m}$ e escala de $0,5 \mathrm{~cm}^{15}$. A medida da circunferência da cintura (CC) foi obtida pela medida da cintura na altura da cicatriz umbilical com fita métrica flexível e inelástica ${ }^{15}$. Para a obtenção da razão CC/Altura foram considerados os valores em centímetros. As medidas das dobras cutâneas tricipital, abdominal, suprailíaca, do peito, subaxilar, subescapular, bicipital, da coxa e da panturrilha medial foram aferidas com adipômetro Cescorf Científico com sensibilidade de $0,1 \mathrm{~cm}$, amplitude de leitura de $88 \mathrm{~mm}$ e pressão de 10 $\mathrm{g} / \mathrm{mm}^{2}$, de acordo com orientação de profissionais da área de Educação Física (www.cdof.com.br). O percentual de gordura corporal (\%G) foi obtido segundo cálculo preconizado por Slaughter et al. ${ }^{16}$, de acordo com o Protocolo para Testes de Avaliação Antropométrica (Percentual de gordura através de medidas de dobras cutâneas), disponibilizado no site www.cdof.com.br.

A medida da pressão arterial foi realizada com os estudantes estando na posição sentada e utilizando-se manguito e esfigmomanômetro. Foi observado repouso inicial de 5 min e foram realizadas duas medidas com intervalo de 5 min. O menor valor encontrado para a pressão arterial foi utilizado no estudo e os resultados foram estratificados de acordo com o sexo, idade e percentil de altura, segundo recomendações da I Diretriz de Prevenção da Aterosclerose na Infância e Adolescência ${ }^{4}$.

\section{Análise Estatística}

Os dados estão apresentados na forma de média e desvio ou mediana e intervalo interquartil. A distribuição dos dados foi avaliada pelo teste de Shapiro-Wilk. As diferenças estatísticas foram detectadas pelo teste $t$ pareado de Student ou teste de Wilcoxon para dados com distribuição normal ou não gaussiana, respectivamente. Todos os testes foram realizados utilizando-se o programa InStat 3.00 (GradhPad - EUA) e MedCalc Statistical Software version 12.7.7 (MedCalc Software bvba, Ostend, Belgium; http://www.medcalc.org; 2013). O valor de $P \leq 0,05$ unilateral foi considerado significativo.

\section{RESULTADOS}


Os valores dos parâmetros bioquímicos e das características biodemográficas dos participantes no início do estudo estão apresentados nas Tabelas 1 e 2, respectivamente (coluna Basal). Com base nos valores do perfil lipídico sérico e da circunferência da cintura, foi observado que $96,2 \%$ dos participantes ( $\mathrm{n}=$ 25) apresentavam pelo menos uma alteração lipídica (dislipidemia) e 80,8\% (n = 21) eram portadores de obesidade abdominal, respectivamente. Além disto, $73 \%$ dos estudantes $(n=19)$ tinham excesso de gordura corporal, avaliada pelo \%G.

TABELA 1 - Valores de parâmetros bioquímicos no período basal e ao final de 3,5 meses de atividades físicas em crianças e adolescentes de Guabiruba-SC, 2011.

\begin{tabular}{lccc}
\hline Parâmetros & Basal & Final & P \\
\hline Perfil lipídico & & & \\
\hline Colesterol Total (mg/dL) & $167,0 \pm 23,8$ & $165,9 \pm 21,1$ & 0,803 \\
\hline LDL-colesterol (mg/dL) & $103,2 \pm 22,6$ & $104,3 \pm 20,9$ & 0,853 \\
\hline HDL-colesterol (mg/dL) & $43,6(38,6-49,3)$ & $45,2(42,3-51,5)$ & 0,006 \\
\hline Não-HDL-colesterol (mg/dL) & $123,4 \pm 25,6$ & $119,4 \pm 22,7$ & 0,340 \\
\hline Triglicérides (mg/dL) & $101,1 \pm 35,9$ & $75,4 \pm 28,8$ & 0,0003 \\
\hline Índice de Castelli I & $3,92 \pm 0,86$ & $3,64 \pm 0,69$ & 0,002 \\
\hline Índice de Castelli II & $2,44 \pm 0,70$ & $2,30 \pm 0,61$ & 0,074 \\
\hline Perfil glicêmico & & & \\
\hline Glicose (mg/dL) & $82,0 \pm 7,8$ & $82,2 \pm 9,5$ & 0,980 \\
\hline Insulina (uUI/mL) & $5,82(2,23-9,90)$ & $3,11(2,01-11,62)$ & 0,280 \\
\hline HOMAi & $1,20(0,46-2,00)$ & $0,69(0,41-2,28)$ & 0,353 \\
\hline Marcadores inflamatórios & & & \\
\hline PCR-as (mg/L) & $0,90(0,53-2,71)$ & $1,36(0,82-2,14)$ & 0,548 \\
\hline Ácido Úrico (mg/dL) & $3,63 \pm 0,61$ & $3,07 \pm 0,69$ & 0,001 \\
\hline
\end{tabular}

Os resultados estão apresentados como média e desvio padrão ou mediana e intervalo interquartil (Percentil 25-75) de 26 indivíduos. Abreviaturas: $\mathrm{HOMAi}=$ Homeostatic model assessment of insulin resistance index; PCR-as = Proteína $\mathrm{C}$ reativa de alta sensibilidade. Teste $t$ pareado de Student ou teste de Wilcoxon.

TABELA 2 - Valores de parâmetros antropométricos e de pressão arterial no período basal e ao final de 3,5 meses de atividades físicas em crianças e adolescentes de Guabiruba-SC, 2011.

\begin{tabular}{lccc}
\hline Parâmetros & Basal & Final & P \\
\hline Peso $(\mathrm{kg})$ & $49,8 \pm 12,8$ & $51,3 \pm 13,0$ & 0,002 \\
\hline Altura $(\mathrm{m})$ & $1,45 \pm 0,09$ & $1,46 \pm 0,09$ & 0,001 \\
\hline Circunferência da Cintura $(\mathrm{cm})$ & $80,8 \pm 12,5$ & $77,3 \pm 12,1$ & 0,001 \\
\hline CC/Altura & $0,56 \pm 0,08$ & $0,53 \pm 0,08$ & 0,001 \\
\hline Percentual de Gordura & $32,6 \pm 9,23$ & $32,3 \pm 9,35$ & 0,726 \\
\hline Pressão Arterial Sistólica & $105,0 \pm 8,6$ & $103,3 \pm 10,6$ & 0,738 \\
\hline Pressão Arterial Diastólica & $60,3(60,0-70,0)$ & $60,0(60,0-60,6)$ & 0,291 \\
\hline
\end{tabular}

Os resultados estão expressos como média e desvio padrão ou mediana e intervalo interquartil (Percentil 25-75) de 26 indivíduos. Abreviaturas: CC = circunferência da cintura. Teste $t$ pareado de Student ou teste de Wilcoxon.

Em comparação com os valores iniciais de parâmetros bioquímicos séricos, foi observado que a prática continuada de atividades físicas recreativas e cooperativas, promoveu redução significativa na concentração de triglicerídeos $(25,4 \%)$, na relação colesterol total/HDL-c (índice de Castelli I) $(7,1 \%)$ e na concentração de ácido úrico (15,4\%). Além disso, houve aumento significativo 
na concentração de HDL-c (3,7\%) (Tabela 1; Figura 1). Em relação ao perfil glicêmico, para 18 estudantes (69,2\%), houve diminuição de 46,5\% (em média) nos valores de insulina $(\mathrm{p}<0,001)$ e de $42,5 \%$ na resistência à insulina (p $<0,001)$. Os demais parâmetros bioquímicos não sofreram modificação significativa pelo exercício físico.
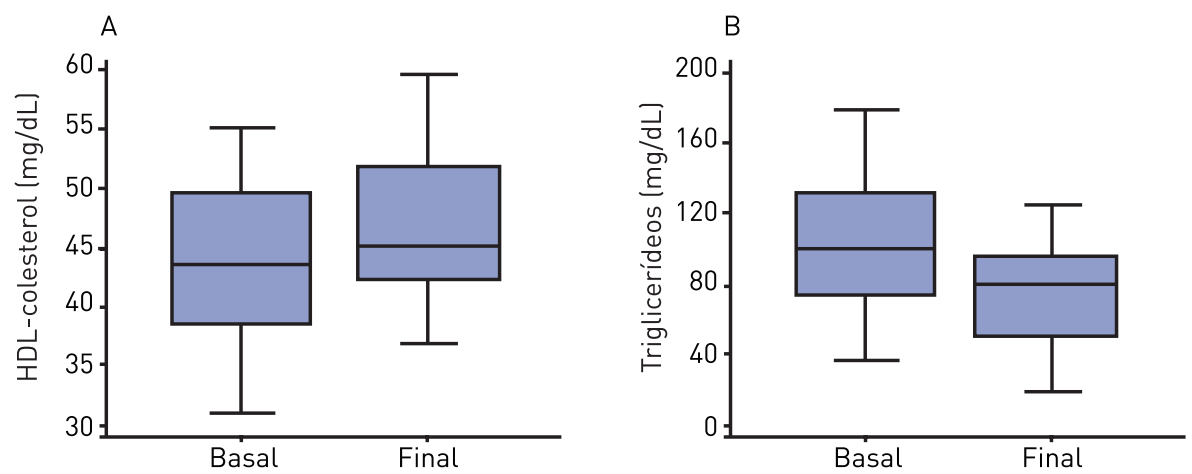

C

D
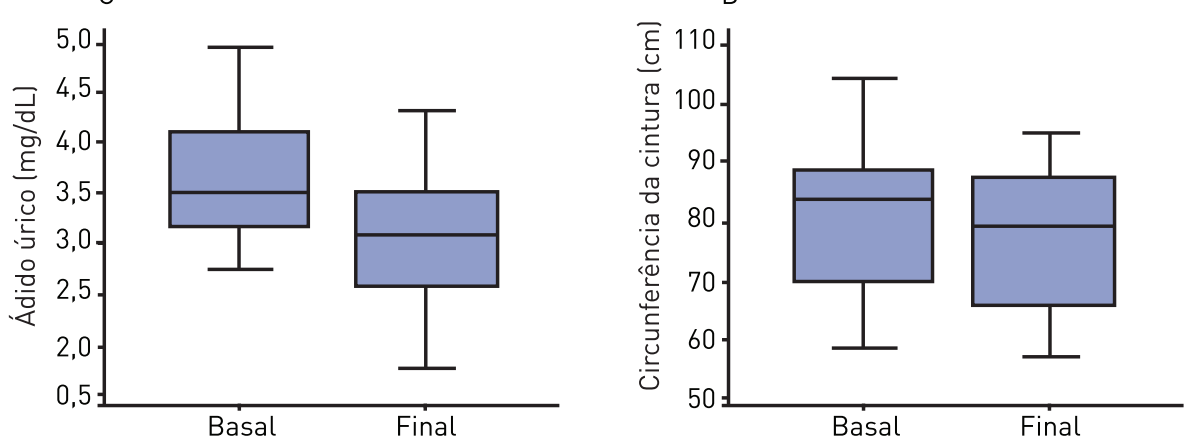

FIGURA 1-Boxplotpara os principaisfatores de riscocardiometabólicoscommelhorassignificativas, reduçõesouaumentos, emseusvaloresmédios.A)HDL-colesterollaumentode 3,7\%);B)Triglicérides (redução de 25,4\%); C) Ácido úrico (redução de 15,4\%); D) Circunferência da cintura (redução de 4,3\%).

Conforme esperado, houve aumento significativo no peso corporal e na altura dos estudantes ao final do estudo ( $\mathrm{p}<0,05$; Tabela 2). Porém, as atividades físicas promoveram redução de $4,3 \%$ na medida da circunferência da cintura e na relação CC/Alt $(5,4 \%)(\mathrm{p}<0,01$; Tabela 2 ; Figura 1$)$. Não houve alteração significativa no percentual de gordura corpórea e na pressão arterial.

\section{DISCUSSÃO}

A importância da atividade física na prevenção das doenças crônicas não transmissíveis, como as DCVs, é de amplo conhecimento e para que este estilo de vida saudável se reflita nos benefícios esperados é fundamental a sua prática de forma regular e consistente ${ }^{17}$. Em adultos, o combate às DCVs consiste na diminuição dos fatores de risco que participam na gênese da desordem sendo importante a precocidade na identificação e consequente redução ${ }^{4}$. Entretanto, ainda permanece em discussão se a diminuição dos fatores de risco em crianças e adolescentes reduzirá a incidência de DCVs na fase adulta.

$\mathrm{O}$ "Physical Activity Guidelines Advisory Committee" ressalta que crianças e jovens que praticam atividade física moderada a vigorosa apresentam perfil de fatores de risco para as DCVs mais favorável em comparação aos jovens sedentários ${ }^{10}$. Entretanto, a relação entre a resposta da atividade física mode- 
rada a vigorosa e a concentração sérica de lipídeos em jovens permanece ainda indeterminada. Além disto, a exata quantidade mínima e ótima de exercícios físicos para a saúde cardiovascular não está estabelecida ${ }^{18}$.

No presente estudo, os valores do perfil lipídico sérico e os índices antropométricos dos participantes mostraram-se mais favoráveis ao final do período de 3,5 meses de atividades físicas recreativas e jogos cooperativos. Ou seja, dentre os parâmetros considerados fatores de risco para as DCVs, a concentração sérica de triglicerídeos e ácido úrico, o índice de Castelli I, a medida da circunferência da cintura e a relação cintura/altura apresentaram redução, enquanto os valores de HDL-colesterol, lipoproteína considerada protetora para as DCVs, aumentou significativamente em relação ao período inicial do estudo. Le Blanc e Jansen ${ }^{19}$ também verificaram que sessões diárias de 15, 30 ou 60 minutos de atividade física moderada a vigorosa, durante quatro ou sete dias, estavam associadas ao aumento de HDL-colesterol e diminuição de triglicerídeos. Ainda corroborando os resultados aqui obtidos, Bezerra et al. ${ }^{20}$ descreveram recentemente em revisão sobre exercícios físicos aeróbicos e perfil lipídico a diminuição significativa de triglicerídeos e aumento de HDL-colesterol. Porém, os autores não encontraram estudos relatando a diminuição do LDL-colesterol, enquanto houve redução de colesterol total em apenas um dos estudos pesquisados. Ressalta-se, entretanto, que as alterações lipídicas observadas por Bezerra et al..$^{20}$ foram obtidas com exercícios físicos de diferentes intensidades e tempo de duração, enquanto no presente estudo foram utilizadas atividades físicas recreativas e jogos cooperativos.

Nesse estudo, não foi observada variação significativa na concentração da glicose de jejum, insulina e resistência à insulina. Porém, ao final do estudo, a atividade física regular promoveu redução significativa de 46,5\% (em média) nos valores de insulina e de 45,9\% na resistência à insulina para 69,2\% (18) dos participantes. Estes resultados são consistentes com o estudo de Bell et al. ${ }^{21}$, nos quais a atividade física promoveu maior sensibilidade à insulina. A redução significativa de triglicerídeos está associada à melhora na sensibilidade à insulina, aumentando o efeito antilipolítico da insulina no tecido adiposo e, consequentemente, diminuindo o transporte de ácidos graxos para o fígado com redução na produção hepática de VLDL-triglicerídeos e de apolipoproteína B-10022. Além disto, a melhora da sensibilidade à insulina aumenta a atividade da enzima lipase lipoproteica, a qual hidrolisa os triglicerídeos das VLDL e quilomícrons no plasma, diminuindo a concentração de triglicerídeos. Concomitantemente, ocorre liberação de fosfolipídeos, colesterol livre e apolipoproteína A da superfície dessas lipoproteínas para dar origem a novas partículas de $\mathrm{HDL}^{23}$, explicando, assim, a elevação na concentração plasmática de HDL-colesterol observada em nosso estudo.

Conforme esperado, o peso corpóreo dos participantes aumentou ao final do período de 3,5 meses de atividades. Porém a adiposidade central, determinada pela medida da circunferência da cintura, e a relação cintura/altura apresentaram diminuição significativa. Até o momento, ainda não há consenso sobre a validade da atividade física para a perda de peso em crianças e adolescentes. Barbeau et al. ${ }^{24}$ observaram redução na gordura corporal em crianças e adolescentes submetidas à prática de exercícios físicos, porém, os resultados de peso corporal e obesidade abdominal foram inconclusivos neste estudo ${ }^{24}$. 
O ácido úrico apresentou redução significativa após a realização das atividades físicas, sugerindo que esse parâmetro pode refletir melhor as alterações inflamatórias em crianças e adolescentes submetidos às atividades físicas.

No presente estudo, incluiu-se o exercício físico aeróbio, associado a jogos de lazer como complemento das atividades físicas. O estudo foi desenvolvido no período extraclasse e o engajamento das atividades fora do horário das aulas contribuiu para o combate ao sedentarismo frequentemente observado neste período. Cabe mencionar, ainda, segundo relato dos participantes, que a inclusão de caminhadas diárias na rotina familiar foram condições somatórias aos processos envolvidos, possivelmente responsáveis pelos resultados obtidos.

O conjunto de atividades desenvolvidas nesse estudo revelou que a atividade física na criança e no adolescente, visando à prevenção dos fatores de risco para as DCVs, não requer a adoção de treinamento intensivo de atividades físicas que exija a capacidade máxima do praticante. Desta forma, o estudo pode ser considerado de baixo custo e de fácil execução e os resultados alcançados são consistentes com as recomendações da International Federation of Sports Medicine $e^{25}$, em que as atividades físicas para crianças sejam aquelas para recreação e educação, não havendo justificativas fisiológicas de treinamento intenso visando à melhora do desempenho para fins competitivos quando o objetivo é a prevenção para as DCVs.

No entanto, vale notar que o pequeno número de indivíduos aqui estudados, o qual não foi representativo da população de estudantes do município de Guabiruba-SC, a falta de avaliação da medida de maturação pubertal e a ausência de grupo controle podem ser considerados como limitação do presente estudo.

Conclui-se, portanto, que a prática de jogos recreativos e cooperativos promoveu melhoras cardiometabólicas nas crianças e adolescentes com dislipidemias e/ou obesidade abdominal que participaram do presente estudo. Com base em nossos resultados, sugere-se que estudos adicionais e de maior amplitude sejam realizados.

\section{Colaboradores}

Nilton Rosini participou na concepção, delineamento, coleta, análise estatística e interpretação dos dados, redação do artigo e aprovou a versão a ser publicada. Rodrigo Diegoli Rosini participou na coleta de dados, concepção e delineamento das atividades físicas e aprovou a versão a ser publicada. Elisa Cristina Vidotto Bruns participou na coleta de dados, acompanhamento nutricional e aprovou a versão a ser publicada. Grasiele Demarche Camillo participou na coleta de dados, concepção e delineamento das atividades físicas e aprovou a versão a ser publicada. Marcos José Machado e Edson Luiz da Silva participaram na concepção, análise, interpretação dos dados e revisão do artigo.

\section{Financiamento}

$\mathrm{O}$ estudo recebeu suporte financeiro da Fundação de Amparo à Pesquisa e Inovação do Estado de Santa Catarina (FAPESC).

\section{Agradecimentos}

Prefeitura Municipal de Guabiruba (Secretaria Municipal de Educação e Secretaria Municipal de Saúde); Secretaria de Estado de Desenvolvimento Regional de Brusque (Gerência de Saúde e Gerência de Educação); FAPESC. 


\section{REFERÊNCIAS}

1. Health statistics and health information systems. 10 facts on the state of global health. Global Health Observatory (GHO). World Health Organization. Disponível em: <www.who.int/publication/en>. Acessado em janeiro, 2012.

2. Ford ES. C-reactive protein concentration and cardiovascular disease risk factors in children: findings from the National Health and Nutrition. Examination Survey 19992000. Circulation. 2003; 108:1053-058.

3. Kwiterovich PO Jr. Recognition and management of dyslipidemia in children and adolescents. J Clin Endocrinol Metab. 2008; 93(11): 4200-4209.

4. Giuliano ICB, Caramelli B, Pellanda L, Duncan B, Mattos S, Fonseca FH. I Diretriz Brasileira de Prevenção da Aterosclerose na Infância e na Adolescência. Arq Bras Cardiol. 2005; 85 Suppl. VI:4-36.

5. Chacra APM, Santos FRD. Dislipidemia em crianças e adolescentes. Rev Soc Cardiol Estado de São Paulo. 2013; 23:71-80.

6. Mota DM, Costa FFF, Azevum A, de Paola AAV, Marin Neto JA. Como melhorar o controle das doenças cardiovasculares? Lições dos estudos epidemiológicos. Rev

7. Soc Cardiol Estado de São Paulo. 2013; 23:14-23.

8. Rosini N, Machado MJ, Webster IZ, Moura SAZO, Cavalcante LS, Silva EL. Simultaneous prediction of hyperglycemia and dyslipidemia in school children in Santa Catarina State, Brazil based on waist circumference measurement. Clin Biochem. 2013; 46: 1837-1841.

9. Rosini N. Perfil de risco cardiovascular em crianças e adolescentes do município de Guabiruba-SC [tese]. Florianópolis (SC): Universidade Federal de Santa Catarina; 2013.

10. Taylor RW, Jones IE, Williams SM, Goulding A. Evaluation of waist circumference, waist-to-hip ratio, and the conicity index as screening tools for high trunk fat mass, as measured by dual energy X Ray absorptiometry, in children aged 3-19y. Am J Clin Nutr. 2000; 72:490-95.

11. Department of Health and Human Service. Physical Activity Guidelines for Americans. Washington, DC, 2008. Disponível em: <http://www.health.gov/paguidelines/>. Acessado em: 07/11/2011.

12. Centers for Disease Control and Prevention. School Healthy Guidelines to Promote Healthy Eating and Physical Activity. Washington, DC, 2011. Disponível em: <http:// www.cdc.gov/healthyyouth/npao/strategies.htm/>. Acessado em: 07/10/2011.

13. Mathwes DR, Hosker JP, Rudenski AS, Naylor BA, Treacher DF, Turner, RC.

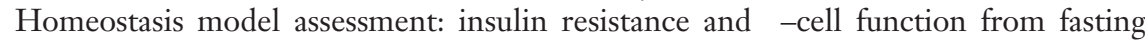
glucose and insulin concentrations in man. Diabetologia. 1985; 28:412-19.

14. Friedewald WT, Levy RI, Fredrickson DS. Estimation of the concentration of lowdensity lipoprotein cholesterol in plasma, without use of the preparative ultracentrifuge. Clin Chem. 1972; 18:499-02.

15. Castelli WP, Abbott RD, McNamara PM. Summary estimates of cholesterol used to predict coronary heart disease. Circulation. 1983; 67:730-34.

16. Alvarez BR, Pavan AL. Alturas e comprimentos. In: Petroski EL, organizadores Antropometria: técnicas e padronizações. Porto Alegre: Pallotti; 2003. p. 29-51.

17. Slaughter MH, Lohman TG, Boileau RA, Horswill CA, Sillman RJ, van Loan MD, et al. Skinfold equations for estimation for body fatness in children and youths. Hum Biol. 1988; 60:709-23.

18. Bracco MM, Carvalho KMB, Baiocchi KM, Bottoni A, Nimer M, Gagliannone CP, et al. Atividade física na infância e adolescência: impacto na saúde pública. Rev Ciências Médicas. 2003; 12:89-97.

19. Physical Activity Guidelines Advisory Committee. Physical Activity Guidelines Advisory Committee Report, 2008. Washington: US. Department of Health and Human Services, 2008.

20. LeBlanc AG, Janssen I. Dose-response relationship between physical activity and dyslipidemia in youth. Can J Cardiol. 2010; 26:e201-e05.

21. Bezerra AIL, Kanegusuku H, Prado WL, Dias RMR, Cardoso Jr CG. Efeito do exercício físico aeróbico e de força no perfil lipídico de seus praticantes: uma revisão sistemática. Rev Bras Ativ Fis Saúde. 2013; 18: 399-411. 
22. Bell LM, Watts K, Siafarikas A, Thompson A, Ratnam N, Bulsara M, et al. Exercise alone reduces insulin resistance in obese children independently of changes in body composition. J Clin Endocrinol Metab. 2007; 92:4230-235.

23. Zanella AM, Souza DRS, Godoy MF. Influência do exercício físico no perfil lipídico e estresse oxidativo. Arq Ciên Saúde. 2007; 14:107-22.

24. Fielding CJ, Fielding PE. Molecular physiology of reverse cholesterol transport. J Lipid Res. 1995; 36:211-28.

25. Barbeu P, Johnson MH, Howe CH, Alisson J, Davis C, Gutin B, et al. Ten months of exercise improves general and visceral, bone, and fitness in black girls. Obesity. 2007; 15: 2077-085.

26. Excessive Physical Training in Children and Adolescents. The International Federation of Sports Medicine. FIMS Position Statement: November 1990. <www.fims.org/ content/assets/documents/PositionStatements/PS5>. Acessado em fevereiro/2012.

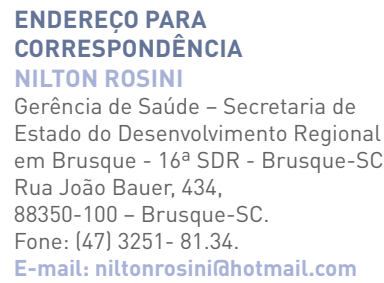

